PROCEEDINGS OF THE

AMERICAN MATHEMATICAL SOCIETY

Volume 131, Number 7, Pages 2255-2259

S 0002-9939(03)06901-6

Article electronically published on February 11, 2003

\title{
THE MODEL CATEGORY OF MAPS OF SPACES IS NOT COFIBRANTLY GENERATED
}

\author{
BORIS CHORNY
}

(Communicated by Paul Goerss)

\begin{abstract}
We show that the model category of diagrams of spaces generated by a proper class of orbits is not cofibrantly generated. In particular, the category of maps between spaces may be supplied with a non-cofibrantly generated model structure.
\end{abstract}

\section{INTRODUCTION AND FORMULATION OF RESULTS}

Many works in homotopical algebra are built on a crucial assumption that a certain model category is cofibrantly generated. For instance, this condition is necessary for the results of P.S. Hirschhorn [12] and J.H. Smith [15] on localization of cellular and combinatorial model categories, respectively. Until recently there was no known example of a non-cofibrantly generated model category and M. Hovey formulated this as an open problem on his home page. Several examples have appeared in response: [1, [4, 9].

In this paper we prove that the model category on diagrams of spaces defined by E. Dror Farjoun in [6] is not cofibrantly generated if the diagrams are of a certain shape $D$. For example, the model category of [6] on the category of maps between spaces is not cofibrantly generated.

We are motivated by the question of existence of localization functors in the above model categories. This question was attacked by V. Halperin in [10, but only the existence of strong localization functors was settled for the equivariant case. The present paper clarifies the conceptual difficulty of this problem, as the general machinery of [12] and [15] is not applicable.

In this paper the category of spaces $\mathcal{S}$ is the category of simplicial sets (or compactly generated topological spaces). If $D$ is a small category, then the category of (D-shaped) diagrams of spaces $\mathcal{S}^{D}$ is the category of functors from $D$ to $\mathcal{S}$ with natural transformations as morphisms. There are many well-known model structures on categories of diagrams of spaces. One of the most widely used is the Bousfield-Kan model category [2], in which the weak equivalences and fibrations are objectwise and the cofibrations are obtained by the left lifting property with respect to trivial fibrations. Another example is given by A. Heller's model category, in which the

Received by the editors February 16, 2002.

2000 Mathematics Subject Classification. Primary 55U35; Secondary 55P91, 18 G55.

Key words and phrases. Model category, equivariant homotopy, non-cofibrantly generated.

The author was a fellow of the Marie Curie Training Site hosted by the Centre de Recerca Matemàtica (Barcelona), grant no. HPMT-CT-2000-00075 of the European Commission. 
weak equivalences and cofibrations are objectwise and the fibrations are obtained by the right lifting property with respect to trivial cofibrations. Heller's model category was used, for instance, by D. Dugger [8] in the proof that every combinatorial model category is Quillen equivalent to one which is simplicial, left proper, and in which every object is cofibrant. These model categories are cofibrantly generated.

We use the word collection to denote a set or a proper class with respect to some fixed universe $\mathfrak{U}$. Let us recall (from [5], 6], 7]) that a $D$-diagram $O$ of spaces is called an orbit if $\operatorname{colim}_{D} O=*$. We denote by $\mathcal{O}_{D}$ the collection of all orbits of $D$ (which is not necessarily a set). Some examples will appear shortly. For any diagram $W$ and a map $f: X \underset{\sim}{Y}$ there is an induced map of spaces $\operatorname{map}(\underline{W}, f): \operatorname{map}(\underline{W}, \underset{\sim}{X}) \rightarrow \operatorname{map}(\underset{\sim}{W} \underset{\sim}{Y}) ;$ see $[5$ for the details.

Definition 1.1. A model category on $\mathcal{S}^{D}$ is generated by the collection $\mathcal{O}_{D}$ of orbits if a morphism $f: \underset{\sim}{X} \rightarrow \underset{\sim}{Y}$ is a

- weak equivalence if and only if $\operatorname{map}(\underset{\sim}{O}, f)$ is a weak equivalence of spaces for any orbit $\underset{\sim}{\sim}$;

- fibration if and only if $\operatorname{map}(\underset{\sim}{O}, f)$ is a fibration of spaces for any orbit $\underset{\sim}{\sim}$;

- cofibration if and only if it has left lifting property with respect to any trivial fibration.

The standard model category axioms were verified in [6]. Functoriality of the factorizations is a recent achievement and will appear in [3]. We use [13] as a reference for the basic facts about model categories. Although the naturality of the factorizations is a part of the axioms in [13], we refer only to the facts which are independent of the functoriality.

The simplest non-cofibrantly generated model category is provided by the following

Theorem 1.2. If $J=(\bullet \rightarrow \bullet)$ is the category with two objects and only one nonidentity morphism, then the functor category $\mathcal{M}=\mathcal{S}^{J}$ of maps of spaces with the model structure generated by the collection of orbits is not cofibrantly generated.

However, not every small category gives rise to a non-cofibrantly generated model category of diagrams. For example, if $D=G$ is a group, then the above model structure on $\mathcal{S}^{G}$ is cofibrantly generated. The crucial difference between the two cases is the "amount" of orbits that depends on the indexing category $D$. If $D=G$ is a group, then the collection of orbits (considered up to $G$-isomorphism) is a set. More precisely, this is the set $\{G / H \mid H \in \Phi(G)\}$, where $\Phi(G)$ is the set of conjugacy classes of subgroups of $G$. In the case of $D=J$ the collection of orbits form a proper class $\{X \rightarrow * \mid X \in \operatorname{obj}(\mathcal{S})\}$.

The paper is organized as follows: after some technical preliminaries in Section 2 we prove in Proposition 3.1 that any model category on diagrams of spaces generated by a proper class of orbits is not cofibrantly generated. Then we deduce Theorem 1.2. In the last section we present many other examples of indexing categories which satisfy the conditions of Proposition 3.1, thus providing more examples of non-cofibrantly generated model categories of diagrams.

\section{Preliminaries}

An orbit over a point $x$ in the colimit of a diagram $\underset{X}{X}$ is the pull back of the canonical map $f: \underset{\sim}{X} \rightarrow \operatorname{colim} \underset{\sim}{X}$ over $x: * \rightarrow \operatorname{colim} \underset{\widetilde{X}}{X}$. Note that every orbit 
over a point is an orbit, in the sense that its colimit is a point. Let $D$ be any small category, and denote by $\mathcal{O}_{D}$ the collection of all orbits of $D$, as above. The operator $\operatorname{codom}(\cdot)$ applied to a collection of maps returns the collection of ranges. Given a set $I$ of maps in $\mathcal{M}=\mathcal{S}^{D}$, we denote by $I$-cell the collection of relative $I$-cellular complexes and by abs- $I$-cell the collection of (absolute) $I$-cellular complexes. See [13, 2.1.9] for precise definitions.

Definition 2.1. Let $\mathcal{X}=\left\{\underline{X}_{\alpha}\right\}_{\alpha \in A}$ be a collection of $D$-shaped diagrams of spaces. The collection of orbits of $\mathcal{X}$, denoted by $\Omega(\mathcal{X})$, consists of all orbits $O_{\alpha, x} \in \mathcal{O}_{D}$ over points $x \in \operatorname{colim} \underset{\alpha}{X_{\alpha}}$, for $\alpha \in A$.

Lemma 2.2. Let $I$ be a set of cofibrations in the model category $\mathcal{M}$ of $D$-shaped diagrams of spaces generated by the orbits. Then $\Omega($ abs- $I$-cell $) \subset \Omega(\operatorname{codom}(I))$.

Proof. Let $X \in \mathcal{M}$ be any $I$-cellular complex. We proceed by transfinite induction on the $I$-cellular filtration of $\underset{X}{X} . \underline{X}_{-1}=\emptyset$. Hence, $X_{0} \in \operatorname{codom}(I)$ and, in particular, $\Omega\left({\underset{\sim}{X}}_{0}\right) \subset \Omega(\operatorname{codom}(I))$.

Suppose ${\underset{\sim}{X}}_{\beta}$ is such that $\Omega\left({\underset{\sim}{X}}_{\beta}\right) \subset \Omega(\operatorname{codom}(I))$. We need to show that ${\underset{\sim}{X+1}}_{\beta+1}$, which is obtained from ${\underset{\sim}{X}}_{\beta}$ by attaching a map $I \ni f: \underset{\sim}{A \hookrightarrow} \underset{\sim}{B}$, satisfies $\Omega\left({\underset{\sim}{\beta+1}}_{\beta}\right) \subset$ $\Omega(\operatorname{codom}(I))$.

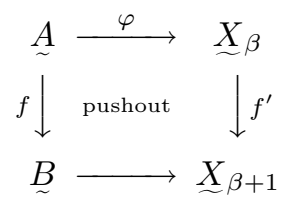

Let $O_{s}$ be an orbit over a point $s \in \operatorname{colim} \underset{\sim}{X+1}=\operatorname{colim} \underset{\sim}{X} \amalg_{\operatorname{colim}} \underset{\sim}{A} \operatorname{colim} \underset{\sim}{B}$. Consider two cases: $s \in \operatorname{colim} \underset{\sim}{X}$ and $s \notin \operatorname{colim} \underset{\alpha_{\beta}}{X}$. In the first case ${\underset{\sim}{s}}_{s}$ is the corresponding orbit of $\underset{X_{\beta}}{X}$ and in the second case ${\underset{\sim}{s}}_{s}$ is some orbit of $\underset{\sim}{B}$. This immediately follows from the fact that the diagrams

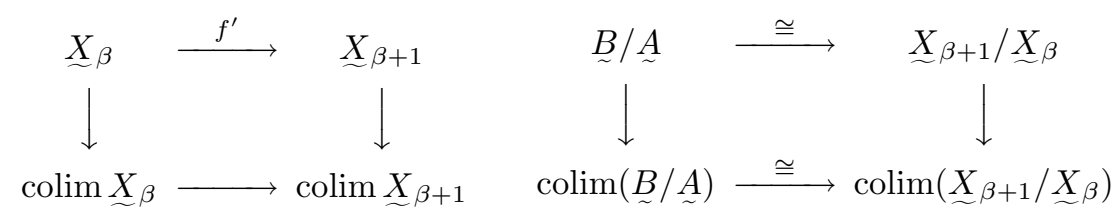

are pull-backs. The first square is a pull-back by [6, 2.1] and the second by the observation that its horizontal maps are isomorphisms. Hence, $\Omega\left({\underset{\sim}{X}}_{\beta+1}\right) \subset \Omega(\operatorname{codom}(I))$.

Obviously, if $\beta$ is a limit ordinal, then

$$
\Omega\left(\underline{X}_{\beta}\right)=\bigcup_{\lambda<\beta} \Omega\left(\underline{X}_{\lambda}\right) \subset \Omega(\operatorname{codom}(I)) .
$$

Hence, $\Omega($ abs- $I$-cell $) \subset \Omega(\operatorname{codom}(I))$.

\section{Proof of Theorem 1.2}

Let us first prove a slightly more general result.

Proposition 3.1. Let $D$ be a small category with a proper class of orbits $\mathcal{O}_{D}$. Then the model category $\mathcal{M}$ on the D-shaped diagrams of spaces generated by the orbits is not cofibrantly generated. 
Proof. We argue by contradiction. Suppose the model category $\mathcal{M}$ generated by the proper class of orbits $\mathcal{O}_{D}$ is cofibrantly generated. If $I$ is the set of generating cofibrations, then any cofibration is a retract of an $I$-cellular map. (This follows from Quillen's small object argument; see [13, 2.1.15].) Then any orbit of $\underset{\sim}{O} \in \mathcal{O}_{D}$ is a retract of an $I$-cellular complex $X$, since any orbit is cofibrant in $\mathcal{M}$. Hence, any orbit is a retract of some orbit ${O_{\sim}^{\prime}}^{\prime}$ over a point in $\underset{\sim}{X}$ :

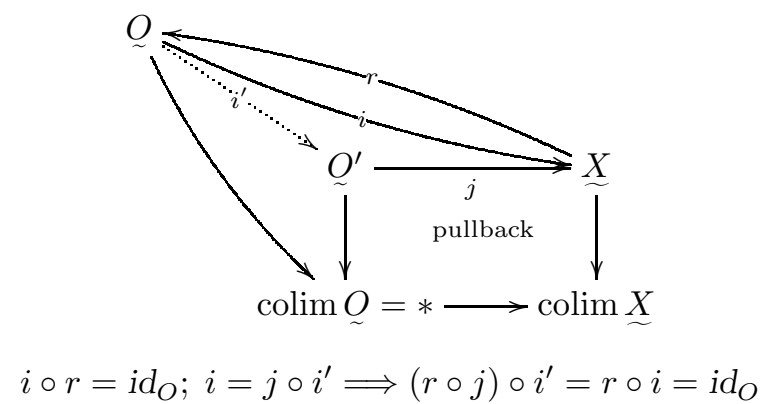

But by Lemma 2.2 the whole collection of orbits of $I$-cellular complexes form a set, hence the contradiction.

The model category on $\mathcal{S}^{J}$ that we are considering is generated by the proper class of orbits $\mathcal{O}_{J}=\{X \rightarrow *\}$, where $X$ runs through all the objects of $\mathcal{S}$. Therefore, by Proposition $3.1, \mathcal{S}^{J}$ is not cofibrantly generated.

\section{More examples}

Let us conclude by giving more examples of non-cofibrantly generated model categories. Proposition [3.1 implies that $\mathcal{M}=\mathcal{S}^{D}$ is not cofibrantly generated iff $\mathcal{O}_{D}$ is a proper class. The following result provides us with a large family of indexing categories which have the required property.

Proposition 4.1. Let $D$ be a small category which admits a fully faithful functor $i: K \rightarrow D$, where $K$ is a category with two objects $k_{1}, k_{2}$, at least one arrow $f: k_{1} \rightarrow k_{2}$ and no arrows in the opposite direction. Then $\mathcal{O}_{D}$ contains a proper class of orbits.

Proof. First for each space $X \in \mathcal{S}$ define an orbit over $K$, i.e. a functor $T_{X}: K \rightarrow \mathcal{S}$, by $\underset{\sim}{T} X\left(k_{1}\right)=X, \underset{\sim}{T} X\left(k_{2}\right)=*, \underset{\sim_{X}}{T}(g)=i d_{X}$ for any $g \in \operatorname{mor}\left(k_{1}, k_{1}\right)$. On the elements of $\operatorname{mor}\left(k_{1}, k_{2}\right)$ and $\operatorname{mor}\left(k_{2}, k_{2}\right), \underset{\sim}{T_{X}}$ has a unique definition, since $*$ is the final object of $\mathcal{S}$. Obviously $\underset{\sim}{T_{X}}$ is an orbit over $K$.

Next for each $\underset{\sim}{T_{X}}$ we define a $D$-orbit $O_{X}$ by extending the definition of $\underset{\sim}{T_{X}}$ to the whole $D$. More precisely, $O_{X}=\operatorname{Lan}_{i}{\underset{\sim}{X}}_{X}$. We need to check that $O_{X}$ is an orbit. This follows from the fact that colimit is itself a left Kan extension along a functor into the trivial category. But any two left Kan extensions commute since they may be represented as coends, and the coends commute by a "Fubini"-type theorem. See [14, X] for the details.

The functor $i$ is fully faithful by assumption, hence $O_{X}\left(i\left(k_{1}\right)\right)=X$. Therefore, we obtain a proper class of $D$-orbits of the form $O_{X}$.

Question 4.2. Let $D$ be a monoid which is not a group. Is $\mathcal{S}^{D}$ cofibrantly generated? 


\section{ACKNOWLEDGMENTS}

The author thanks C. Casacuberta and E. Dror Farjoun for helpful conversations about the subject matter of this paper.

\section{REFERENCES}

[1] J. Adámek, H. Herrlich, J. Rosický and W. Tholen, Weak factorization systems and topological functors, preprint, 2000.

[2] A.K. Bousfield and D.M. Kan, Homotopy Limits, Completions and Localizations, Lecture Notes in Math. 304, Springer-Verlag, 1972. MR 51:1825

[3] B. Chorny, Localization with respect to a class of maps, in preparation.

[4] D. Christensen and M. Hovey, Quillen model structures for relative homological algebra, preprint, 2001.

[5] E. Dror Farjoun, Homotopy and homology of diagrams of spaces, Proceedings of the Conference on Algebraic Topology, Seattle 1985, Lecture Notes in Math. 1286, 93-134, SpringerVerlag, 1987. MR 89b:55012

[6] E. Dror Farjoun, Homotopy theories for diagrams of spaces, Proc. Amer. Math. Soc. 101, 181-189, 1987. MR 88h:55013

[7] E. Dror Farjoun and A. Zabrodsky, Homotopy equivalence between diagrams of spaces, J. Pure Appl. Alg. 41, 169-182, 1986. MR 87g:55021

[8] D. Dugger, Combinatorial model categories have presentations, preprint, 2001. MR 2002k: 18022

[9] D. Isaksen, A model structure on the category of pro-simplicial sets, Trans. Amer. Math. Soc. 353, no. 7, 2805-2841, 2001. MR 2001m:18015

[10] V. Halperin, Equivariant Localization of Diagrams, Ph.D. thesis, Hebrew University, Jerusalem, 1998.

[11] A. Heller, Homotopy theories, Mem. Amer. Math. Soc. 71, no. 383, 1988. MR 89b:55013

[12] P.S. Hirschhorn, Localization of Model Categories, preprint, April 12, 2000.

[13] M. Hovey, Model Categories, Mathematical Surveys and Monographs vol. 63, Amer. Math. Soc., 1999. MR 99h:55031

[14] S. MacLane, Categories for the Working Mathematician, Graduate Texts in Math. 5, Springer-Verlag, 1971. MR 50:7275

[15] J.H. Smith, Combinatorial Model Categories, unpublished.

Einstein Institute of Mathematics, Givat Ram, The Hebrew University of Jerusalem, JERUSALEM 91904, ISRAEL 